\title{
Recherche \\ clinique et action \\ humanitaire
}

> Dans les années 1990 et face à l'absence de données, Médecins sans frontières (MSF) a mis en place des études cliniques afin de mesurer l'efficacité des antipaludéens et d'adapter les traitements au sein de ses missions. Entre 1996 et 2004, plus de 12000 patients furent inclus dans 43 études réalisées dans 18 pays d'Asie et d'Afrique. Ces études ont été conduites dans des zones d'accès difficile et d'insécurité, en général dans des pays d'Afrique subsaharienne. Elles ont permis d'améliorer la qualité des traitements dans les missions de MSF, de contribuer à adapter les protocoles nationaux dans certains pays et d'influencer les changements des recommandations au niveau international. Le nombre important de publications scientifiques issues des missions de MSF montre qu'une recherche clinique de qualité peut être conduite malgré les nombreuses difficultés rencontrées sur le terrain. Ces études nécessitent une rigueur suffisante pour que les résultats soient valides scientifiquement, mais elles doivent aussi pouvoir bénéficier de la souplesse requise compte tenu des conditions de terrain particulières. Bien que ce travail démontre le rôle potentiel des ONG (organisations non gouvernementales) médicales dans la collecte de données scientifiques, la responsabilité première dans le lancement de telles études devrait revenir aux ministères de la santé, à l'OMS (Organisation mondiale de la santé) et à d'autres organismes internationaux en charge de la lutte contre les maladies endémiques. <

Médecins sans Frontières (MSF) est une organisation humanitaire qui apporte des soins médicaux à des populations dans des contextes de guerre, de famine, d'épidémies, de catastrophes naturelles ou de déplacement de populations. Au cours des années 1990, la résistance a à la chloroquine ( $\mathrm{CQ}$ ) et à la sulphadoxinepyriméthamine (SP), les médicaments utilisés généra-

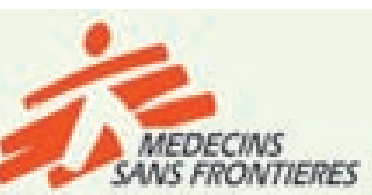

Ancien responsable de la recherche sur le paludisme à Épicentre. Actuellement : Responsable du Programme de couverture vaccinale, Unité Maladies à Prévention Vaccinale, Département de Maladies Infectieuses, Institut de veille sanitaire, 12 , rue du Val d'Osne, 94415 Saint-Maurice, France. jp.guthmann@invs.sante.fr

lement pour traiter le paludisme simple à Plasmodium falciparum $(P f)$, affectait déjà les populations de la plupart des pays d'intervention de MSF, principalement en Afrique. Cette résistance était considérée comme responsable d'une importante morbi-mortalité par paludisme $[1,2]$. Cependant, les données scientifiques documentant cette résistance étaient relativement limitées, si bien que ces médicaments continuaient à être recommandés par les gouvernements de ces pays et par l'OMS (organisation mondiale de la santé). Les combinaisons à base d'artémisinine (artemisinin-based combination therapies, ou ACT), utilisées en Asie du sud-est depuis le début des années 1990, apparaissaient comme la meilleure alternative à ces médicaments de première (CQ) et de seconde (SP) lignes [3]. Vers la fin des années 1990, l'absence de données fiables sur l'ampleur de la crise d'efficacité des antipaludéens d'une part, et le manque de financements et de réactivité de la part de la plupart des organismes internationaux de contrôle et de recherche d'autre part laissaient présager un véritable désastre sanitaire [3]. Dans ce contexte, et confronté dans le travail quotidien des missions à des échecs thérapeutiques aux conséquences parfois fatales, MSF se lança alors dans une série d'études d'efficacité in vivo, avec le soutien d'Epicentre (association satellite de MSF consacrée à la recherche opérationnelle et à l'épidémiologie d'intervention) et en collaboration avec les ministères de la santé nationaux. 
Ces études, utilisant les protocoles recommandés par l'OMS, eurent comme principal objectif de documenter la situation et d'adapter les traitements au sein des programmes de MSF.

Après avoir exposé brièvement les principaux résultats obtenus au cours de ces études réalisées entre 1994 et 2004, nous discutons leurs conséquences sur les stratégies de traitement du paludisme et essayons de tirer quelques leçons de cette expérience, décrite avec plus de détail dans le numéro d'août 2008 de la revue PLoS Medicine [4].

\section{Principaux résultats}

Entre 1994 et 2004, 43 études cliniques ont été conduites dans 18 pays, dont huit ( $17 \%$ ) en Asie (Laos, Myanmar (3) ${ }^{1}$, Bangladesh (2), Inde, Cambodge) et 35 ( $83 \%$ ) en Afrique (Nigeria (2), Libéria (2), Sierra Leone (3), Mali, Guinée, Tchad (2), Ouganda (5), Sud Soudan (4), Nord Soudan, RDC (6), Congo, Zambie, Angola (5), Burundi). Au cours de ces études, plus de 12000 patients ont été recrutés et 117 types de traitements ont été analysés: 69 (59\%) étaient des monothérapies [CQ (chloroquine), sulphadoxine-pyriméthamine (SP), amodiaquine (AQ), méfloquine $(M Q)], 43(37 \%)$ des ACT (artésunate + SP, CQ ou $\mathrm{AQ}$; artéméther + luméfantrine ; dihydroartémisinine + pipéraquine) et 5 (4\%) d'autres combinaisons (par exemple CQ + SP).

\section{Contribution à la recherche}

Lorsque l'on évalue la part prise par les études effectuées par MSF parmi toutes les études de ce type réalisées dans ces mêmes pays pendant cette période, elles représentent $25 \%$ du total (112 types de traitements - monothérapies et ACT - évalués par MSF sur un total de 455) (Tableau I). La plus grande contribution est celle des études effectuées dans des pays instables d'Afrique subsaharienne, sièges de conflits armés, comme l'Angola, le Burundi, le Tchad, le Liberia, le Sierra Leone ou le Soudan. Dans ces pays dans lesquels les institutions académiques nationales ou internationales ne sont généralement pas (ou très peu) présentes, ces données représentent la très grande majorité des données disponibles. Les articles publiés par MSF (31/137, $23 \%$ ) représentaient environ le quart des articles publiés sur le même sujet dans ces mêmes pays (Tableau II). Ces résultats montrent qu'une recherche clinique de qualité peut être réalisée malgré les difficultés techniques et logistiques rencontrées sur le terrain.

\section{Contribution aux changements des politiques de traitement dans les pays d'intervention}

Les données sur l'efficacité des antipaludéens issues des études de MSF ont d'abord permis à l'association de mieux traiter les patients sur ses terrains d'intervention. De façon plus générale, elles ont aussi parfois contribué à adapter les protocoles nationaux de traitement du paludisme en fonction des données locales et nationales même

\footnotetext{
${ }^{1}$ Le chiffre entre parenthèses indique le nombre d'études lorsqu'il y en a plus d'une dans un même pays.
}

lorsque ces études ont été menées dans des zones spécifiques (conflits armés, déplacements de population, crises nutritionnelles) ne permettant pas d'extrapoler les résultats à l'ensemble du pays (on sait par exemple que les enfants malnutris ont probablement un risque plus élevé d'échec thérapeutique $[5,6]$ ). En effet, les recommandations effectuées par MSF à l'issue de ces études ont été le plus souvent (12 pays sur 18 , soit $66 \%$ ) cohérentes avec les changements de politique de traitement antipaludéen décidés ultérieurement nationalement (Tableau 1). Cela suggère que ces résultats furent considérés comme des arguments significatifs dans les décisions politiques adoptées par les gouvernements de ces pays. Cette allégation doit néanmoins être affirmée avec prudence tant il est vrai que les facteurs menant à un changement de politique sanitaire, comme les raisons conduisant au choix d'un traitement plutôt qu'un autre, sont nombreux, complexes et difficiles à mesurer.

Le rôle de ces études dans l'adaptation des protocoles de traitement intervenue à l'échelle de certains pays peut s'expliquer, tout au moins en partie, par la période historique au cours de laquelle ces études ont été menées (principalement au début des années 2000), époque charnière où de nombreux pays pensaient encore que la chloroquine et la SP étaient efficaces et où l'OMS, en dépit de données disponibles de plus en plus nombreuses, ne recommandait pas encore l'utilisation des ACT (recommandés en 2003 [7]). Quelques exemples illustrent comment, sur la base d'une expérience de terrain et des données scientifiques produites, MSF fut amené à jouer un rôle de conseil influençant les décisions prises plus tard par certains gouvernements en matière de lutte contre le paludisme. Au Burundi, les études menées pendant l'importante épidémie de paludisme de 2000-2001 [8] montrèrent que chloroquine et SP n'étaient pas ou peu efficaces. Compte tenu de ces résultats, des discussions furent instituées avec les autorités du pays et avec I'OMS qui aboutirent finalement à l'adoption de la combinaison artésunate + amodiaquine en juillet 2002 par les autorités nationales. Dans d'autres pays, le processus de changement fut moins controversé. Après les études au Sierra Leone, une réunion de consensus fut organisée par le Ministère de la santé de ce pays en mars 2004 avec la participation du gouvernement central, des autorités sanitaires régionales, de l'OMS, des bailleurs et des ONG. Au cours de cette réunion, les résultats obtenus par MSF furent discutés et un consensus en faveur de l'utilisation des ACT fut obtenu. Une conférence du même type fut aussi organisée par MSF à Khartoum (Soudan) en 


\begin{tabular}{|c|c|c|c|c|c|c|c|}
\hline \multirow[t]{2}{*}{ Pays } & \multicolumn{2}{|c|}{$\begin{array}{l}\text { Etudes MSF/ } \\
\text { Total d'études }\end{array}$} & \multirow[t]{2}{*}{$\begin{array}{l}\text { Année(s) } \\
\text { de l'étude }\end{array}$} & \multirow[t]{2}{*}{$\begin{array}{c}\text { Recommandation } \\
\text { MSF }\end{array}$} & \multirow[t]{2}{*}{$\begin{array}{c}\text { Année } \\
\text { de changement }\end{array}$} & \multirow[t]{2}{*}{$\begin{array}{l}\text { Nouvelle } \\
\text { politique }\end{array}$} & \multirow[t]{2}{*}{$\begin{array}{l}\text { Concordance } \\
\text { recommandation } \\
\text { MSF et nouvelle } \\
\text { politique }\end{array}$} \\
\hline & $\mathrm{n} / \mathrm{N}$ & $\%$ & & & & & \\
\hline \multicolumn{8}{|l|}{ Asie } \\
\hline Bangladesh & $2 / 6$ & 33 & 2003 & $A S-M Q, A-L$ & 2004 & $A-L$ & Oui \\
\hline Cambodge & $2 / 17$ & 12 & 2003 & Aucune & 2000 & AS-MP & Non \\
\hline Inde & $8 / 42$ & 19 & 2001 & AS-MP & 2004 & SP, AS-SP & Non \\
\hline Laos & $1 / 13$ & 8 & 2000 & Aucune & 2001 & $A-L$ & Non \\
\hline Myanmar & $10 / 73$ & 14 & $1998,2001-04$ & $A S+M Q$ & 2002 & AS-MQ, A-L & Oui \\
\hline Total & $23 / 151$ & 15 & & & & & \\
\hline \multicolumn{8}{|l|}{ Afrique } \\
\hline Angola & $12 / 20$ & 60 & $1997,2003-04$ & $A S-A Q, A-L$ & 2004 & $A-L$ & Oui \\
\hline Burundi & $4 / 12$ & 33 & 2001 & $A S-A Q, A-L$ & 2003 & $A S-A Q$ & Oui \\
\hline Congo & $3 / 8$ & 37 & 2004 & $A-L, A S-A P$ & Pas de changement & & Non \\
\hline $\mathrm{RDC}$ & $14 / 33$ & 42 & 2001-04 & $A-L$ & 2004 & AS-AQ & Non \\
\hline Guinée & $2 / 10$ & 20 & 2004 & $A S-S P, A S-A Q$ & 2004 & AS-AQ & Oui \\
\hline Mali & $2 / 22$ & 9 & 2004 & AS-SP, AS-AQ, A-L & 2004 & $A-L$ & Oui \\
\hline Nigéria & $2 / 20$ & 10 & 2001-02 & ACT, Non précisée & 2004 & $A-L$ & Oui \\
\hline Liberia & $3 / 3$ & 100 & 2002 & AS-AP & 2004 & AS-AP & Oui \\
\hline S. Léone & $16 / 18$ & 89 & 2001-03-04 & AS-AQ & 2004 & AS-AQ & Oui \\
\hline Soudan & $12 / 50$ & 24 & 2001-03 & AS-SP, A-L & 2004 & $A S-A Q, A S-S P$ & Oui \\
\hline Tchad & $6 / 7$ & 86 & 2002-03 & AS-AP & 2004 & AS-AQ & Oui \\
\hline Ouganda & $11 / 54$ & 20 & $1998,2000-02-03-04$ & AS-AQ, AS-SP, A-L & 2004 & $A-L$ & Oui \\
\hline Zambie & $2 / 47$ & 4 & 2002 & $A-L$ & 2002 & $A-L$ & Oui \\
\hline Total & $89 / 304$ & 29 & & & & & \\
\hline TOTAL & $112 / 455$ & 25 & & & & & \\
\hline
\end{tabular}

Tableau I. Nombre d'études réalisées par MSF/Épicentre parmi toutes les études par pays et type de traitement (monothérapies et ACT) et concordance entre les recommandations de MSF et la nouvelle politique adoptée ultérieurement par le pays [4]. A-L: artemether-luméfantrine; AS: artésunate; SP : sulfadoxine-pyriméthamine; AP : amodiaquine ; MQ : méfloquine ; CQ : chloroquine.

octobre 2003, qui réunit des experts internationaux ainsi que de nombreux professionnels du pays directement impliqués dans la lutte contre le paludisme. Elle aboutit à l'adoption de la combinaison artésunate + SP dans les zones du pays contrôlées par le gouvernement de Khartoum et fut suivie d'une réunion similaire convoquée par l'OMS en janvier 2004 à Nairobi (Kenya) avec les représentants du sud Soudan.

\section{Leçons tirées de cette expérience}

\section{Intégration au sein d'une mission} humanitaire et difficultés logistiques

La conduite de cette recherche dans certains terrains isolés s'est souvent heurtée à de nombreuses difficultés scientifiques, logistiques ou liées à l'adaptation d'une équipe de recherche dans une mission médicale. Bien que réalisée en collaboration avec Epicentre, organisation créée dans la 


\begin{tabular}{|c|c|c|}
\hline & Nombre total des articles & Nombre d'articles MSF \\
\hline Angola & 3 & 3 \\
\hline Libéria & 2 & 2 \\
\hline Sierra Léone & 2 & 2 \\
\hline Tchad & 2 & 2 \\
\hline Bangladesh & 7 & 2 \\
\hline Burundi & 3 & 0 \\
\hline Ouganda & 24 & 4 \\
\hline Soudan & 15 & 4 \\
\hline Myanmar & 5 & 3 \\
\hline Guinée & 1 & 1 \\
\hline $\mathrm{RDC}$ & 2 & 2 \\
\hline Cambodge & 5 & 1 \\
\hline Inde & 15 & 1 \\
\hline Laos & 9 & 1 \\
\hline Congo & 5 & 1 \\
\hline Mali & 4 & 1 \\
\hline Nigéria & 27 & 0 \\
\hline Zambie & 6 & 1 \\
\hline Total & 137 & $31(23 \%)$ \\
\hline
\end{tabular}

Tableau II. Nombre et proportion d'articles publiés dans des revues à comité de lecture, 1996-2007 [4].

deuxième moitié des années 1980 et dont l'expertise en épidémiologie d'intervention dans des terrains difficiles est reconnue, l'évaluation de l'efficacité des antipaludéens était quelque chose de nouveau à MSF. Même si ces études ont été menées parallèlement aux programmes de soins, leur intégration au sein d'une mission humanitaire a du être acceptée par les équipes soignantes. La logistique des missions a dû être adaptée et une logique de recherche (avec ses contraintes méthodologiques et sa rigueur) introduite dans des missions composées en grande partie par des cliniciens dont l'objectif principal est de soigner des malades. Cependant, cette intégration s'est faite le plus souvent sans problème majeur et la bonne disposition des équipes sur place a été probablement un élément déterminant pour mener ces études jusqu’à leur terme.

Outre ces difficultés internes, des contextes d'intervention particulièrement difficiles nécessitant une logistique complexe ont pu parfois ralentir la réalisation de ces études. À Caala, ville dévastée par la guerre en Angola, la population était affectée par une urgence nutritionnelle pendant la période d'étude et le climat de grande tension qui y régnait ainsi que les actions destinées en priorité à la prise en charge des enfants dénutris n'étaient pas favorables à la conduite de l'étude. À
Bundibugyo, camp de déplacés à l'ouest de l'Ouganda, l'incursion répétée de rebelles anti-gouvernementaux a nécessité la mise en place de mesures spécifiques pour le suivi des patients, y compris des visites à domicile par les cliniciens et le personnel de laboratoire se rendant souvent à pied dans des lieux situés à plusieurs kilomètres de marche de la clinique. La plupart des sites du Sud Soudan n'étaient accessibles qu'en petit avion et leur accès était en grande partie dépendant des aléas de la météorologie empêchant parfois un atterrissage sur une piste boueuse. Des laboratoires ont dû être entièrement mis sur pied dans des lieux sans électricité, impliquant le transport de microscopes, de centrifugeuses, de réfrigérateurs et de générateurs. Du personnel paramédical et de laboratoire, souvent absent des zones d'intervention en raison de l'effondrement du système sanitaire, a dû parfois être amené à partir d'autres endroits du pays. Lorsque cela n'a pas été possible, des professionnels étrangers ont dû être recrutés spécifiquement pour participer à l'étude. Toutes ces circonstances ont conduit MSF et Epicentre à être flexibles et innovants et à s'adapter à des situations toujours nouvelles, parfois changeantes au cours d'une même étude. Dans ce processus et dès lors que MSF avait fait le choix de mener ces études, le soutien politique et les importants moyens matériels et humains mis en œuvre ont été essentiels à leur succès.

\section{Stratégie de recherche et qualité des données}

Les études réalisées par MSF n'ont pas été toujours systématiquement planifiées selon un calendrier de recherche précis, comme peuvent l'être des programmes de recherche scientifique plus classiques, mais plutôt décidées en fonction des circonstances et de la situation locale. Ces circonstances ont parfois conduit, nous l'avons dit, à des adaptations aux contraintes du terrain. La mise en place de ces études dans des régions instables où le risque d'être confronté à la nécessité d'une évacuation était omniprésent est à ce titre instructif. Au début, compte tenu de ce risque, il a été décidé d'une allocation séquentielle des traitements, par exemple traitement initial d'un groupe de patients par chloroquine, puis une fois le premier groupe terminé, traitement d'un deuxième groupe par chloroquine + artésunate. Au fil des mois, ayant constaté que ces évacuations restaient tout de même exceptionnelles, les patients ont été comparés en assurant une allocation randomisée, bien plus rigoureuse scientifiquement. Plus tard et afin de ne pas perdre du temps en mettant en place un processus d'évaluation trop long, il a été décidé de comparer directement deux ACT en faisant l'hypothèse (non nécessairement démontrée dans le pays) que la monothérapie était moins efficace. 
En même temps que nous adoptions une attitude plus pragmatique au risque d'altérer la rigueur scientifique, des efforts étaient réalisés pour améliorer la qualité de ces études. Un guide et des procédures standardisées ont été rédigés qui pouvaient être utilisés sur tous les terrains. Un double contrôle microscopique des frottis de sang a été mis en place afin de réduire les erreurs de lecture. Un suivi plus strict des patients a permis de mieux détecter les effets secondaires des traitements évalués et les éventuelles complications, et aussi de minimiser le nombre de «perdus de vue »; ce suivi, souvent de 14 jours initialement, a été plus tard étendu à 28 jours, durée considérée comme minimale pour mesurer plus justement l'efficacité des anti-paludéens. Enfin, la technique de génotypage du plasmodium par PCR a été systématiquement mise en place, ce qui a permis de différencier les souches plasmodiales résistantes de celles entraînant de nouvelles infections et donc d'obtenir des résultats plus fiables et plus faciles à interpréter. Ce génotypage a d'ailleurs nécessité la mise en place de collaborations avec des institutions (le plus souvent, mais pas toujours, européennes) qui, en raison de leur importante charge de travail, ont parfois fourni les résultats avec des délais considérables. Dans ces situations, les résultats finaux ont été rendus avec un retard de plusieurs mois, responsable d'une position inconfortable vis-à-vis des autorités sanitaires du pays et même des équipes soignantes de MSF qui réclamaient ces résultats pour adapter le traitement localement. Plus tard, le développement d'un laboratoire à Mbarara (Ouganda) en collaboration avec l'université de cette ville, a permis en partie de réduire cette dépendance et de raccourcir les délais dans le rendu des résultats.

\section{Questions d'éthique}

Au cours de ces études, MSF et Epicentre ont été confrontés à certaines questions d'éthique communes à la réalisation de protocoles de recherche dans les situations de crise humanitaire [9]. Par exemple, même si les cliniciens sur le terrain avaient le plus souvent la conviction que les traitements utilisés étaient peu efficaces, nous avons rarement disposé de données scientifiques pour soutenir ces affirmations. Ce manque d'information de base constituait objectivement un frein à la promotion de nouvelles thérapeutiques; malgré cela, de nouveaux traitements ont quand même été testés. D'autre part, en raison de leurs conditions de vie difficiles et des difficultés d'accès au système de soins, les populations n'ont pas toujours eu un vrai choix de participer à ces études. Le site de l'étude était souvent le seul lieu offrant à ces patients la possibilité de se soigner et même si une information détaillée leur était donnée par une personne de leur communauté et dans leur langue, leur compré- hension a pu dans certains cas être insuffisante pour considérer objectivement les risques et les bénéfices encourus lors de leur participation à une telle étude. Enfin, dans un grand nombre de pays, il n'existait pas de comité d'éthique, le protocole était alors simplement approuvé par le ministère de la santé et par les autorités régionales.

Dans toutes ces situations, il a été considéré que collecter des données sur l'(in)efficacité des antipaludéens était essentiel, non seulement pour les patients localement mais plus généralement pour stimuler un changement des stratégies de traitement du paludisme qui, rappelons le, était loin d'être promu par l'OMS et d'autres grandes institutions et experts internationaux au début des années 2000. La position de MSF était motivée par les arguments suivants: les protocoles d'évaluation utilisés étaient ceux recommandés par l'OMS; les régimes testés n'étaient pas expérimentaux; enfin, le risque encouru par un patient enrôlé dans l'étude était inférieur à celui d'un patient traité en routine dans le service de santé local en raison d'un suivi rigoureux et systématique et de la possibilité d'utiliser à tout moment des traitements de seconde ligne efficaces. Dans ces conditions, il a été considéré que la balance «bénéfice-risque » penchait en faveur du patient.

Cette expérience suggère que dans ces situations, les risques éthiques dans la conduite d'une recherche devraient toujours être évalués en tenant compte des conséquences possibles d'un statu quo. Les risques doivent être minimisés par la standardisation des protocoles, la rédaction de procédures de consentement bien comprises et adaptées au contexte local, le développement de structures alternatives qui puissent donner un avis et une validation, enfin la participation de la communauté au processus de réalisation de l'étude et à la prise des principales décisions.

\section{Conclusions}

Ces études sont indispensables pour adapter les outils thérapeutiques (ou diagnostiques) dans les missions de MSF. Elles nécessitent une rigueur suffisante pour que les résultats soient valides sur le plan scientifique, mais doivent aussi pouvoir bénéficier de la souplesse requise par des conditions de terrain difficiles. Bien que ce travail démontre le rôle potentiel des ONG médicales dans la collecte de données scientifiques, la responsabilité première dans le lancement de telles études devrait revenir aux ministères de la santé, à l'OMS et à d'autres partenariats internationaux en charge de la lutte contre les maladies endémiques. $\diamond$

\section{SUMMARY}

Clinical research and humanitarian work:

the role of Médecins sans Frontières in the fight against malaria

In the 1990s and in the absence of data, Médecins sans frontiers (MSF) initiated a series of in vivo clinical studies to measure antimalarial efficacy and optimise treatment strategies within its missions. Between 1996 and 2004, more than 12.000 patients were enrolled in 43 studies in 18 countries of Asia and Africa. These studies were conducted in insecure and difficult-to-access sites, usually conflict-affected countries of subSaharan Africa. The data were used by MSF to improve treatments within its missions. In several countries they were considered by health authorities for adapting treatment guidelines at a country level. They had an effect 
on international policy. The high number of publications in peer-reviewed journals shows that research of high quality can be performed despite the important logistical difficulties encountered in the field. These studies are essential to adapt treatments for MSF programs. They need to be rigorous enough to be scientifically valid but also have to be adapted to specific field conditions. Although this work demonstrates the potential role of medical NGOs (non governmental organisations) such as MSF in collecting scientific data, national malaria programs, WHO and other international disease control partnerships hold the primary responsibility in initiating such studies. $\diamond$

\section{REMERCIEMENTS}

Nous remercions Pascal Ringwald (Global Malaria Programme) WHO) de nous avoir communiqué les données compilées par l'OMS. Nous remercions également les nombreux auteurs et institutions ayant participé à chacune de ces études, qui malheureusement ne peuvent être cités dans cet article. Merci à Francesco Checchi (OMS, Genève) et à Marc Gastellu-Etchegorry, vice-président de la section française de MSF (Institut de Veille Sanitaire, France) pour avoir bien voulu apporter des commentaires à ce manuscrit. Merci à Nick White (Wellcome Trust), François Nosten (Wellcome Trust) et Piero Olliaro (TDR-WHO) pour leur soutien et leur enseignement au cours de ces années.

\section{TIRÉS À PART}

J.P. Guthmann

\section{LISTE DES PRINCIPAUX INVESTIGATEURS DE CHA९UE ÉTUDE INDIVIDUELLE:}

C. Bachy, M. Bonnet, P. Campbell, F. Checchi, દ. Depoortere, X. de Radiguès, F. Grandesso, J. P. Guthmann, B. Janssens, S. Hamour, R. Hardwick, D. Legros, P. Piola, T. Prinsberg, G. Priotto, F. Smithuis, દ. Stivanello, T. Swarthout, I. van den Broek.

\section{NOTE (DISCLAIMER)}

L'auteur a coordonnée la recherche sur l'efficacité des antipaludéens entre 2000 et 2006. Les opinions exprimées dans cet article ne reflètent pas nécessairement celles de «Médecins sans Frontières ».

\section{RÉFÉRENCES}

1. Trape JF. The public health impact of chloroquine resistance in Africa. Am J Trop Med Hyg $2001 ; 64$ (suppl 1/2) : 12-7.

2. Zucker JR, Ruebush II TK, Obonyo C, et al. The mortality consequences of the continued use of chloroquine in Africa: experience in Siaya, Western Kenya. Am J Trop Med Hyg 2003; $68: 386-90$.

3. White NJ, Nosten F, Looareesuwan S, et al. Averting a malaria disaster. Lancet 1999 ; 353: 1965-7.

4. Guthmann JP, Checchi F, van den Broek I, et al. Assessing antimalarial efficacy in a time of change to artemisinin-based combination therapies: the role of Médecins Sans Frontières. PLoS Med 2008 ; 5 : el69.

5. Hess FI, lannuzzi A, Leafasia J, et al. Risk factors of chloroquine resistance in Plasmodium falciparum malaria. Acta Trop 1996; 61: 293-306.

6. Hamel MJ, Holtz T, Mkandala C, et al. Efficacy of trimethoprim-sulfamethoxazole compared with sulfadoxine-pyrimethamine plus erythromycin for the treatment of uncomplicated malaria in children with integrated management of childhood illness dual classifications of malaria and pneumonia. Am J Trop Med Hyg $2005 ; 73$ : 609-15.

7. World Health Organisation. Position of WHO's roll back malaria. Department on malaria treatment policy. Genève : WHO, 2003.

8. Guthmann JP, Bonnet M, Ahoua L, et al. Deaths rates from malaria epidemics, Burundi and Ethiopia. Emerg Infect Dis $2006 ; 13: 140-3$.

9. Committee on Population National Research Council Research. Ethics in complex humanitarian emergencies. Summary of a workshop. Washington, DC : National Academy Press, 2002.

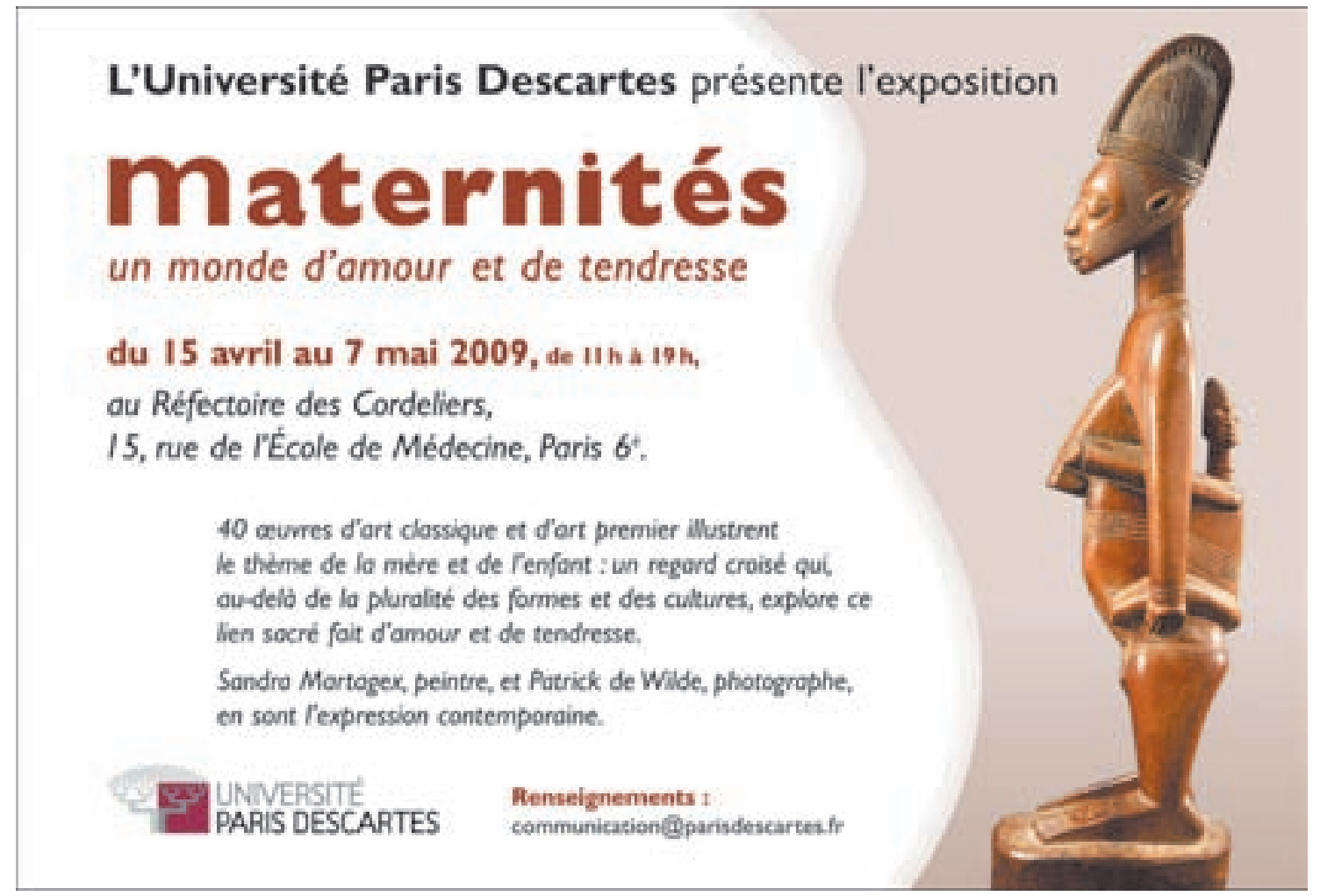

